Pesq. Vet. Bras. 29(11):905-909, novembro 2009

\title{
Afecções podais em vacas da bacia leiteira de Rondon do Pará1
}

\author{
José Alcides S. Silveira ${ }^{*}$, Tatiane T. Albernaz ${ }^{2}$, Carlos Magno C. Oliveira² ${ }^{2}$ \\ Marcos D. Duarte ${ }^{2}$ e José Diomedes Barbosa ${ }^{2}$
}

\begin{abstract}
Silveira J.A.S., Albernaz T.T., Oliveira C.M.C., Duarte M.D. \& Barbosa J.D. 2009. [Foot disorders in cows from basin milk of Rondon do Pará.] Afecções podais em vacas da bacia leiteira de Rondon do Pará. Pesquisa Veterinária Brasileira 29(11):905-909. Central de Diagnóstico Veterinário, Universidade Federal do Pará, Rua Maximino Porpino da Silva 1000, Pirapora, Castanhal, PA 68743-080, Brazil. E-mail: jalcides@ufpa.br

Foot diseases are some of the main causes of low performance in dairy cows. In order to determine the prevalence, clinical classification and to establish the epidemiological factors of foot diseases in cows from basin milk of Rondon do Pará, 1,236 cows were evaluated and 275 had at least one type of foot injury. A total of 655 lesions was showed which a prevalence of $22.25 \%$. Most frequent lesions were interdigital hyperplasia (80.92\%), claw overgrowth (6.42\%), and interdigital necrobacillosis $(6.11 \%)$. Hind limbs were most frequently affected $(61.83 \%)$, and lesions affecting the interdigital space were the most common in both the thoracic (36.34\%) and hind limbs (48.09\%). Epidemiological study showed that the environmental characteristics such as mountainous areas, newly formed grassland (with trunks and branches of trees), irregular ground, and mud and gravel in the floor of the pens may promote the development of foot lesions. In $95.5 \%$ of the farms, no measures had been taken for the control and prophylaxis of foot diseases. The specific clinical examination of the feet was efficient for the diagnosis.
\end{abstract}

INDEX TERMS: Dairy cows, foot diseases, Pará.

RESUMO.- As doenças digitais estão entre as principais causas de performance reduzida de rebanhos leiteiros. Com o objetivo de determinar a prevalência, classificar clinicamente e estabelecer os fatores epidemiológicos das enfermidades podais em vacas da bacia leiteira de Rondon do Pará, foram avaliadas 1.236 vacas, das quais 275 apresentaram pelo menos um tipo de lesão podal. Identificouse 655 lesões, o que resultou em uma prevalência de $22,25 \%$. As enfermidades mais frequentes foram hiperplasia interdigital $(80,92 \%)$, necrobacilose interdigital $(6,11 \%)$ e cascos com crescimento excessivo $(6,42 \%)$.

\footnotetext{
${ }^{1}$ Recebido em 23 de maio de 2009.

Aceito para publicação em 24 de junho de 2009.

Parte da Dissertação de Mestrado do primeiro autor, Curso de PósGraduação em Ciência Animal, Núcleo de Ciências Agrárias e Desenvolvimento Rural, Universidade Federal do Pará (UFP), Empresa Brasileira de Pesquisa Agropecuária - Amazônia Oriental, Universidade Federal Rural da Amazônia.

${ }^{2}$ Central de Diagnóstico Veterinário, UFP, Rua Maximino Porpino da Silva 1000, Pirapora, Castanhal, PA 68743-080, Brasil. "Autor para correspondência: jalcides@ufpa.br
}

Os membros pélvicos foram os mais acometidos (61,83\%) e o espaço interdigital, tanto nos membros torácicos $(36,34 \%)$, quanto nos pélvicos $(48,09 \%)$, a região digital acometida com maior frequência. O estudo epidemiológico mostrou que características ambientais tais como relevo montanhoso, pastagem em formação com presença de troncos e galhos de árvores, irregularidades nos pisos dos currais, presença de piçarra e lama podem favorecer o aparecimento das lesões podais. Constatou-se a ausência de medidas de controle e profilaxia de afecções que acometem os cascos em $95,5 \%$ das propriedades estudadas. O exame clínico específico do casco foi eficiente no diagnóstico das enfermidades.

TERMOS DE INDEXAÇÃO: Vacas leiteiras, afeç̧ões podais, Pará.

\section{INTRODUÇÃO}

Estudos prévios em outras regiões do Brasil associaram altas prevalências de afecções podais em bovinos com rebanhos submetidos ao manejo intensivo em instalações com pisos de cimento e acúmulos de fezes e urina, bem 
como à alimentação inadequada e à ausência de medidas de controle (Cruz et al. 2001a, 2001b, Dias et al. 2001, Martins et al. 2002, Ferreira et al. 2004). Embora frequentemente manejados em condições mais naturais (sistema extensivo ou semi-intensivo) com prolongado acesso a áreas de pastagens, estudos preliminares em rebanhos bovinos do Pará, tanto de leite quanto corte, também registraram doenças digitais em prevalências consideráveis (Silveira et al. 2008). À semelhança do que foi observado previamente (Cruz et al. 2001a), aquele estudo identificou aspectos adicionais relacionados à importância dessas doenças tais como número de lesões por animal, de animais acometidos por propriedade e gravidade das lesões que comprometem diferentes estruturas do casco. Altas prevalências dessas lesões no Brasil, como em outros países, têm tradicionalmente sido associadas com aspectos ambientais que submetem os dígitos a traumas ou condições de umidade e anaerobiose, conforme observado em Minas Gerais (Molina et al. 1999), Goiás (Silva 2001), Rio Grande do Sul (Cruz et al. 2001a, 2001b) e Mato Grosso do Sul (Martins et al. 2002). Embora consideráveis perdas econômicas tenham sido observadas nesses estudos, variações significativas foram registradas quanto às prevalências das diferentes lesões digitais, provavelmente devido aos diferentes sistemas de produção, níveis de manejo sanitário, condições climáticas regionais e constituições raciais dos rebanhos, entre outros fatores. Este estudo registra a prevalência das afecções podais em rebanhos de bovinos leiteiros do estado do Pará e descreve alguns fatores epidemiológicos associados com sua ocorrência.

\section{MATERIAL E MÉTODOS}

O estudo foi realizado em rebanhos leiteiros de propriedades localizadas na bacia leiteira de Rondon do Pará e abrangeu os municípios de Rondon do Pará, Abel Figueiredo e Bom Jesus do Tocantins, onde foram avaliadas vacas em lactação. Inicialmente, as vacas foram avaliadas clinicamente em posição quadrupedal, em estação e em movimento. Quando detectada, claudicação de qualquer intensidade e/ou lesão digital visível, realizou-se a contenção para exame clínico específico dos cascos. As vacas eram derrubadas através do método Italiano, o qual auxiliava o posicionamento dos mesmos em decúbito lateral sobre o solo, e após eram contidas com cordas. Em seguida, os dígitos eram limpos com água e escova. Após esse preparo, realizava-se o exame específico dos dígitos através da inspeção, palpação direta, palpação indireta e percussão (Dirksen 1993). As afecções foram classificadas conforme descrições apresentadas anteriormente (Weaver et al. 1981, Greenough et al. 1983, Hull et al. 1993, Venter \& Amstel 1994) e avaliadas através de um protocolo de dados para diagnóstico de claudicação em bovinos, de acordo com Greenough et al. (1997). Os fatores epidemiológicos foram registrados durante inspeção das pastagens, instalações e vias de acesso, os quais os bovinos percorriam durante o manejo diário. Paralelamente, foi conduzido um questionário epidemiológico a fim de determinar os fatores predisponentes ao aparecimento das afecções podais.

\section{RESULTADOS}

\section{Epidemiologia}

Foram estudadas 22 propriedades, onde foram examinadas 1.236 vacas leiteiras, das quais 275 apresentaram lesões (prevalência de 22,25\%). Essas vacas eram mestiças zebuínas, predominantemente da raça Indubrasil, em fase de lactação e com idade entre dois e 15 anos. As propriedades visitadas empregavam manejo em regime extensivo e possuíam solos arenosos e áreas com relevos montanhosos. Havia pastagem em formação com presença de troncos e galhos de árvores (Fig.1-2) em sete fazendas $(31,8 \%)$ e pastagens estabelecidas há mais de cinco anos em quinze (68\%). As pastagens, em todas as propriedades, eram constituídas principalmente de Brachiaria brizantha e os bovinos recebiam mineralização em cochos, à vontade. Em dezesseis fazendas (72,7\%) foram verificados currais sem cobertura, com pisos irregulares de chão batido, às vezes, calçados com cascalho (piçarra) (Fig.3-4). A higienização, quando feita, apenas incluía raspagem. Na maioria das vezes, havia pedras,

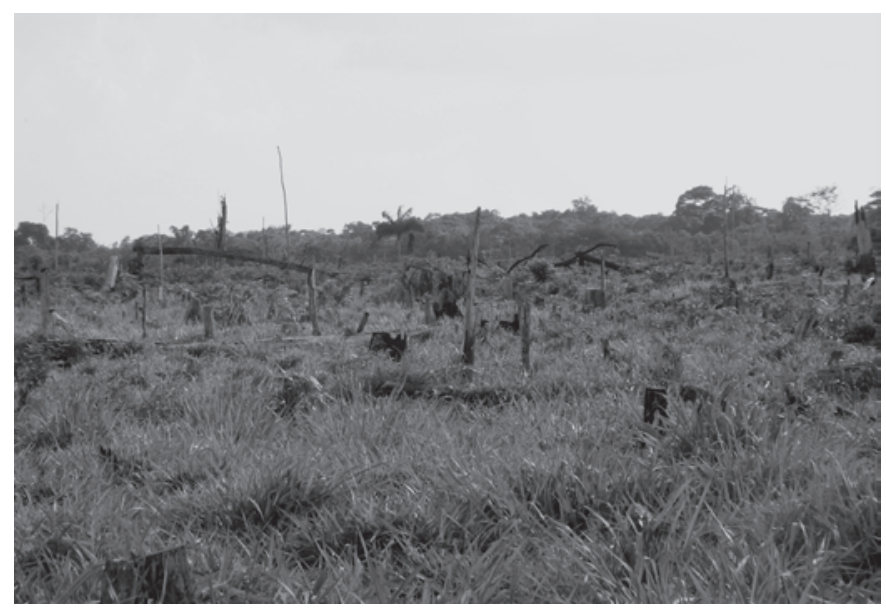

Fig.1. Pasto recém-formado de Brachiaria brizantha contendo troncos e galhos de árvores.

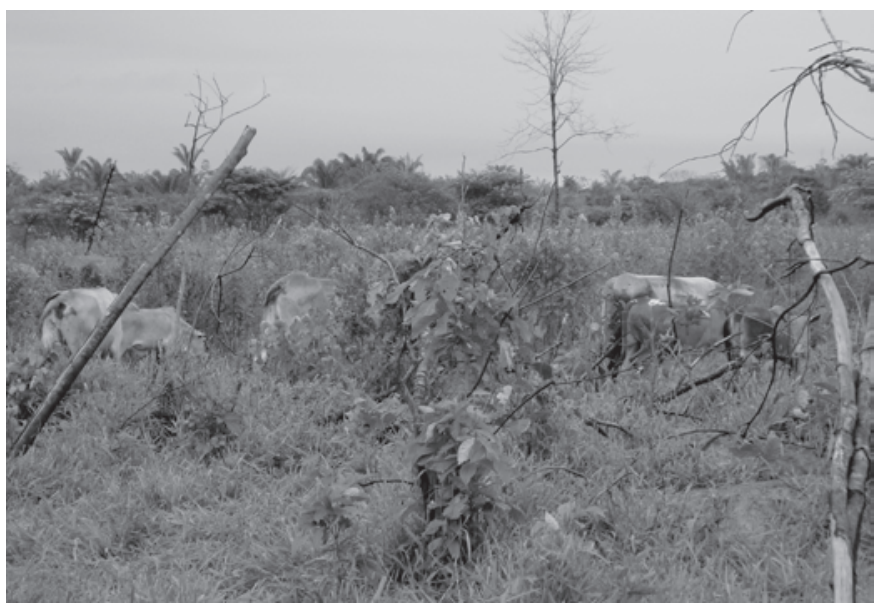

Fig.2. Bovinos em pastagem recém-formada de Brachiaria brizantha contendo troncos e galhos de árvores. 


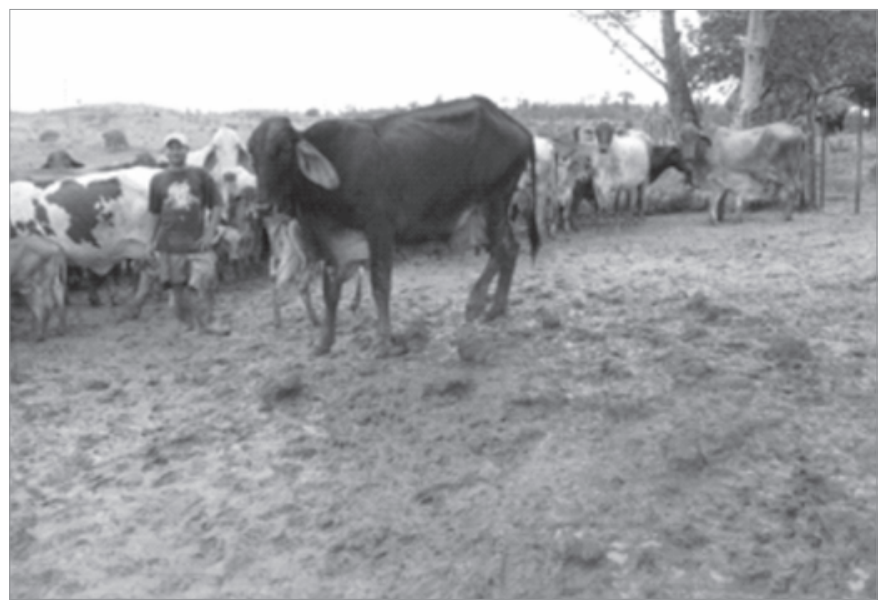

Fig.3. Estábulo sem cobertura, com piso irregular, contendo cascalho (piçarra), lama, fezes e urina.

Fig.4. Estábulo sem cobertura, com piso irregular.

Fig.5. Acúmulo de lama no entorno dos currais, onde as vacas permanecem na espera de serem ordenhadas.

além de outros materiais traumatizantes e acúmulo de matéria orgânica e lama (Fig.5) nos currais de espera.

\section{Características clínicas}

A maioria das vacas apresentava lesões visíveis nos cascos sem claudicação. Vacas com lesões mais graves apresentavam claudicações de graus I a V, emagrecimento, alterações de posturas, anestro prolongado e diminuição na produção de leite, além de sinais sugestivos de deficiências minerais como osteofagia, emagrecimento progressivo e perda de coloração em áreas da pelagem. As lesões podais diagnosticadas nesse estudo estão apresentadas no Quadro 1. As lesões de hiperplasia interdigital foram as mais frequentes e se caracterizavam pela proliferação da pele do espaço interdigital, na maioria das vezes, presente em mais de um membro, com intensidade entre
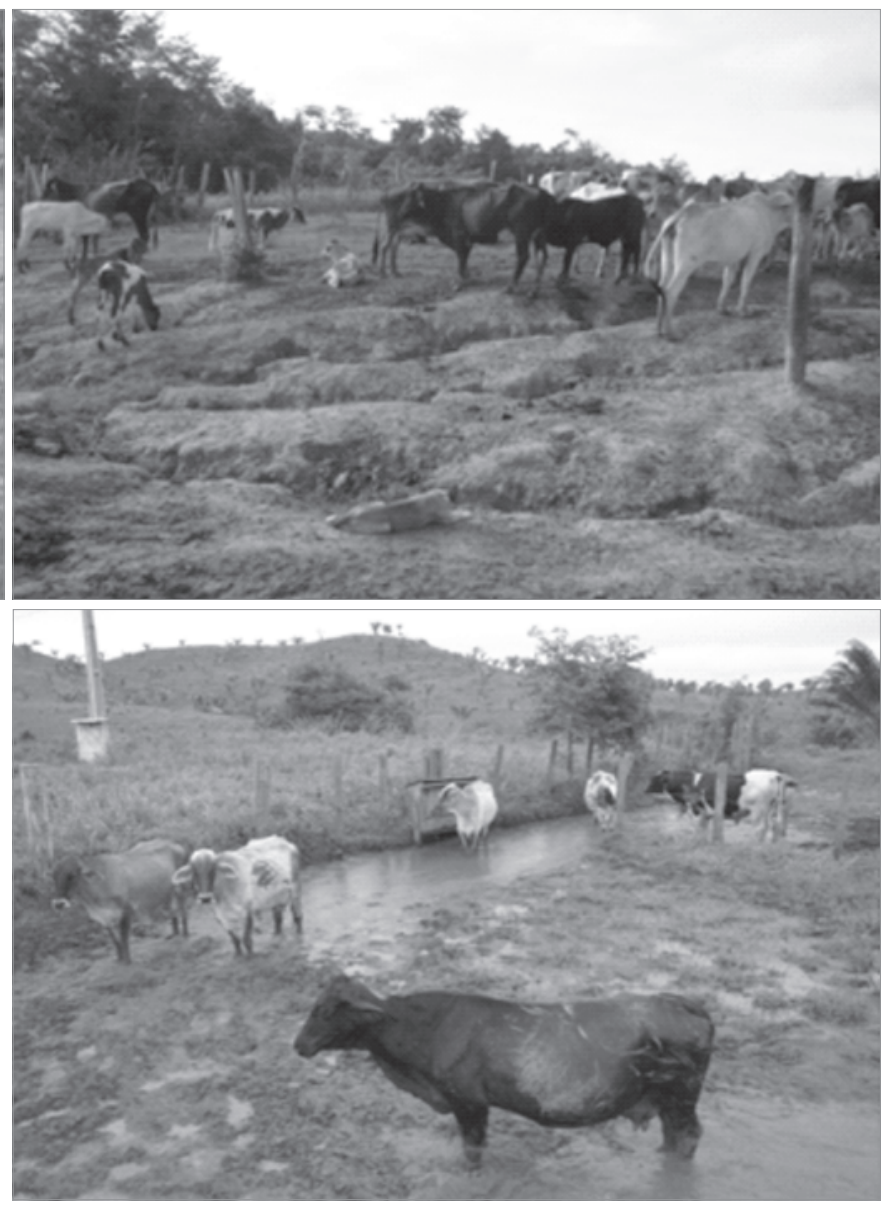

leve (sem claudicação) e grave (com ulceração e claudicação) (Fig.6). A necrobacilose interdigital se caracterizava pela perda de tecido córneo associada com necrose e/ou abscesso solar e tecido de granulação no espaço interdigital e áreas ulceradas da sola, muralha ou talão, na maioria das vezes, secundária à hiperplasia interdigital. Os crescimentos excessivos dos cascos se caracterizavam por deposições córneas excessivas nos talões e paredes abaxiais
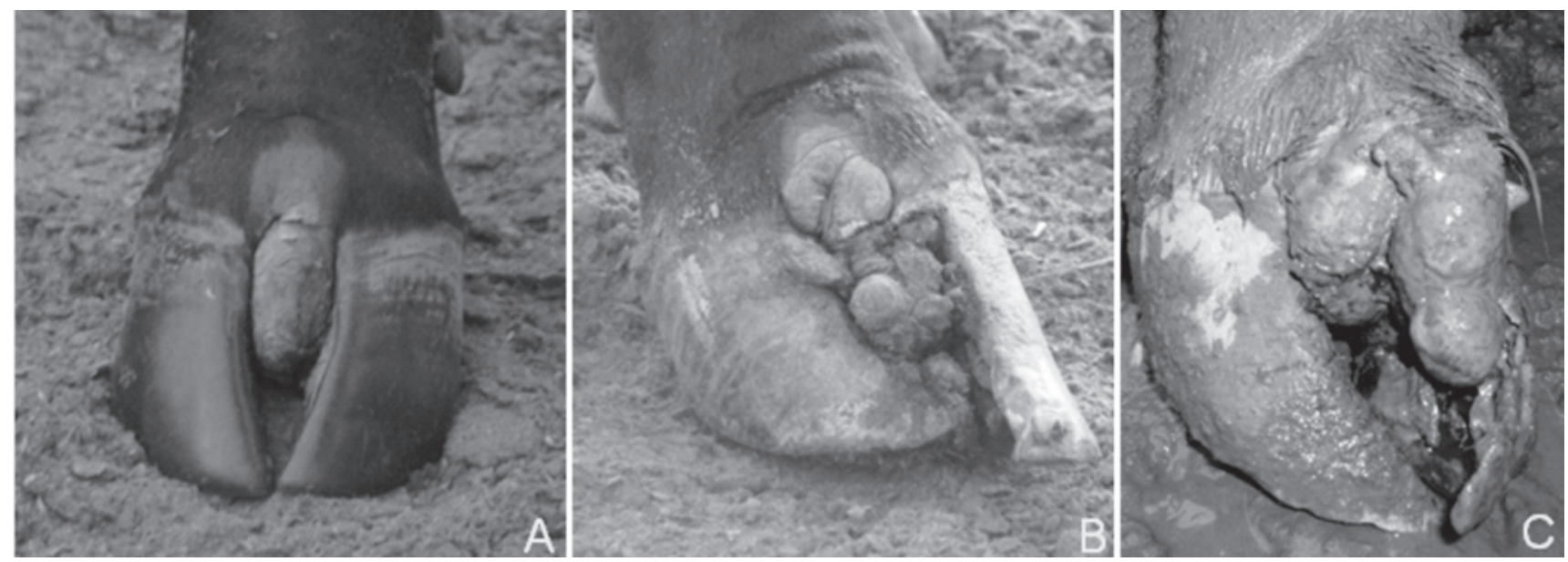

Fig.6. (A) e (B) Hiperplasia interdigital em diferentes estágios de evolução. (C) Complicação de hiperplasia interdigital, com tecido de granulação e perda de tecido córneo. 


\section{Quadro 1. Prevalência de afeç̧ões podais diagnosticadas em 275 de 1.236 vacas leiteiras examinadas na bacia leiteira do município de Rondon do Pará}

\begin{tabular}{lcc}
\hline Afecções podais & Número de lesões & $\%$ \\
\hline Hiperplasia interdigital & 530 & 80,92 \\
Crescimento excessivo do casco & 42 & 6,42 \\
Necrobacilose interdigital & 40 & 6,11 \\
Pododermatite - unhas acessórias & 16 & 2,44 \\
Erosão de talão & 6 & 0,92 \\
Dermatite digital & 6 & 0,92 \\
Casco achinelado & 4 & 0,61 \\
Artrite séptica interfalangeana distal & 3 & 0,46 \\
Dermatite interdigital & 3 & 0,46 \\
Sola dupla & 3 & 0,46 \\
Casco em forma de saca rolha & 1 & 0,15 \\
Úlcera de pinça & 1 & 0,15 \\
Totais & 655 & 100
\end{tabular}

caudais, além do comprimento exagerado das pinças (Fig.6B). Os membros pélvicos foram os mais acometidos $(61,83 \%)$ e o espaço interdigital, tanto nos membros torácicos $(36,34 \%)$, quanto nos pélvicos $(48,09 \%)$, a região digital acometida com maior frequência.

\section{DISCUSSÃO}

As lesões digitais mais frequentes neste estudo foram os fibromas ou hiperplasias interdigitais. Essas lesões são reações proliferativas, com hiperplasia do tecido fibroso denso da pele interdigital e tecido subcutâneo que ocorrem em resposta à irritação crônica decorrente de traumas ou condições ambientais, de acúmulo de dejetos e excessiva umidade (Weaver et al. 1981, Hull et al. 1993). Neste sentido, pastos altos, morros, restos de árvores nas pastagens, currais com pisos contendo cascalho, lama e altos índices pluviométricos foram provavelmente os fatores predisponentes mais importantes da condição descrita aqui. Aspectos semelhantes foram verificados anteriormente (Cruz et al. 2001a, Silva 2001, Ferreira 2003, Borges et al. 2002, Borges \& Pitombo 2007). Além disso, a manutenção de estábulos e piquetes em condições adequadas para locomoção segura dos animais é fator importante para o equilíbrio entre desgaste e crescimento dos cascos (Dirksen \& Stöber 1981, Moraes 2000). O estiramento da pele interdigital que pode ser decorrente de conformação anormal do dígito, mau manejo de correção de cascos, condições ambientais ou predisposição hereditária pode causar a proliferação. Um significante número de fibromas não está associado à claudicação; entretanto, quando infectados, ulcerados ou traumatizados, têm alta probabilidade de causarem manqueira (Weaver et al. 1981, Hull et al. 1993, Venter \& Amstel 1994). As vacas com lesões graves e claudicação, também apresentaram emagrecimento, anestro prolongado, diminuição na produção de leite e deficiências nutricionais. Déficit de condição corporal (Hassal et al. 1993) e déficit no desempenho reprodutivo (Melendez et al. 2002, Gabarino et al. 2004) têm sido observações comumente associadas com animais com claudicação.
Este estudo demonstrou predomínio absoluto da hiperplasia interdigital $(80,92 \%)$; entretanto, também houve associações relevantes com necrobacilose e crescimentos excessivos. Embora as prevalências dessas lesões estivessem mais equilibradas em estudos anteriores (Cruz et al. 2001, Martins et al. 2002, Silveira et al. 2008), todas são condições determinadas por fatores predisponentes comuns, principalmente aspectos ambientais e/ou decorrentes do manejo inadequado (Hull et al. 1993, Venter \& Amstel 1994). No nosso país, a hiperplasia interdigital foi associada com relevo montanhoso (Borges et al. 2002, Martins et al. 2002), mas também com a irritação decorrente dos acúmulos de dejetos e lama (Cruz et al. 2001a). O efeito exercido pelo capim sobre o espaço interdigital, especialmente em animais que têm que percorrer longas distâncias em terrenos com relevos acentuados, tem sido associado com a patogenia da hiperplasia interdigital (Welker 1993). Além disso, a predisposição genética dos mestiços zebuínos parece estar ligada também ao excesso de tecido adiposo interdigital (Borges \& Pitombo 2007). A segunda alteração em prevalência foi crescimento excessivo do casco $(6,42 \%)$, deformidade córnea frequentemente associada à ocorrência prévia de acidose ruminal e laminite ou falta de desgaste (Toussaint Raven 1985, Allestein 1994). Essa última mais provavelmente foi a causa nos bovinos deste estudo, uma vez que permanecem a maior parte do tempo em pastagens e regulam sua ingestão de fibras, diminuindo sobremaneira os riscos de acidose. Além disso, as alterações de postura consequentes de outras lesões contribuem para o crescimento excessivo dos cascos (Toussaint Raven 1985). A terceira lesão podal de maior ocorrência foi a necrobacilose ou pododermatite séptica difusa $(6,11 \%)$, a qual foi, na maioria das vezes, complicação secundária da hiperplasia interdigital e provavelmente ocorreu por falta ou atraso no tratamento (Dirksen et al. 2005).

A presença das dermatites digitais neste estudo constitui aspecto digno de nota, uma vez que essa é uma condição associada com epidemiologia semelhante (Weaver et al. 1981, Hull et al. 1993, Venter \& Amstel 1994) à observada neste estudo e inclusive previamente identificada no Brasil (Cruz et al. 2001b, Martins et al. 2002). Entretanto, como esta é uma condição mais comumente observada em médios e grandes rebanhos leiteiros manejados em sistema intensivo ou de semi-confinamento, é possível que tais variações provavelmente sejam determinadas por associações multifatoriais que incluam além dos fatores ambientais, outros de origem nutricional, genética (Allestein 1994, Venter \& Amstel 1994) e especialmente microbiana (Cruz et al. 2005).

Agradecimentos.- À Fundação de Amparo a Pesquisa do Estado do Pará (FAPESPA Edital no 04/2007), pelo apoio financeiro. Aos proprietários das fazendas estudadas, pela contribuição na realização deste estudo.

\section{REFERÊNCIAS}

Allenstein L.C. 1994. Distúrbios da locomoção dos bovinos. Simpósio Internacional sobre Produção Intensiva de Leite. Interleite, São Paulo, p.53-65. (Resumo) 
Borges J.R., Pitombo C.A. \& Mársico Filho F. 2002. Revisão de 31 casos cirúrgicos de hiperplasia interdigital em bovinos. Revta Bras. Med. Vet. 24:10-15.

Borges J.R.J. \& Pitombo C.A. 2007. Doenças digitais de etiologia incerta ou secundárias: pododermatite do paradígito, p.526. In: Riet-Correa F., Schild A.L., Lemos R.A.A. \& Borges J.R.J. (Eds), Doenças de Ruminantes e Eqüídeos. Vol.2. 3aㅡ ed. Palotti, Santa Maria.

Cruz C.E.F., Driemeier D., Cerva C. \& Corbellini L.G. 2001a. Clinical and epidemiological aspects of bovine digital lesions in southern Brazil. Arq. Bras. Med. Vet. Zootec. 53(6):654-657.

Cruz C.E.F., Driemeier D., Cerva C. \& Corbellini L.G. 2001b. Bovine digital dermatitis in southern Brazil. Vet. Rec. 148:576-577.

Cruz C.E.F., Pescador C., Nakajima Y. \& Driemeier D. 2005. Immunopathological investigations on bovine digital epidermitis. Vet. Rec. $157: 834-840$

Dias R.O.S \& Marques A.P. 2001. Casco em Bovinos. Lemos Editorial, São Paulo, p.37-41.

Dirksen G. \& Stöber E.M. 1981. As afecções dos cascos dos bovinos: melhor prevenir que curar. Hora Vet., Porto Alegre, 1(3):13-18.

Dirksen G., Gründer H.D. \& Stöber M. 1993. Rosenberger's Exame Clínico dos Bovinos. $3^{\text {a }}$ ed. Guanabara Koogan, Rio de Janeiro, p.322326.

Dirksen G., Gründer H.D. \& Stöber M. 2005. Medicina Interna y Cirurgía del Bovino. 4ª ed. Inter-Médica, Buenos Aires, p.826-901.

Ferreira P.M. 2003. Enfermidades podais em rebanho leiteiro confinado. Tese de Doutorado em Medicina Veterinária, Escola Veterinária, Universidade Federal de Minas Gerais, Belo Horizonte, MG. 79p.

Ferreira P.M., Leite R.C., Carvalho A.V., Facury Filho E.J., Souza R.C. \& Ferreira M.G. 2004. Custo e resultados do tratamento de seqüelas de laminite bovina: relato de 112 casos em vacas em lactação no sistema free-stall. Arq. Bras. Med. Vet. Zootec. 56(5):589-594.

Gabarino E.J., Hernandez J.A. \& Shearer J.K. 2004. Effect of lameness on ovarian activity in postpartum holstein cows. J. Dairy Sci. 87:41234131.

Greenough P.R., McCallum F.I. \& Weaver A.D. 1983. Les Boiteries des Bovins. $3^{\text {ième }}$ ed. Du Point Veterinaire, Paris, p.151-218.

Greenough P.R., Weaver A.D., Broom D.M., Esslemont R.J. \& Galindo F.A. 1997. Basic Concepts of Bovine Lameness. $3^{\text {rd }}$ ed. W.B. Saunders, Philadelphia, p.3-13.

Hassal S.A., Ward W.R. \& Murray R.D. 1993. Effect of lameness on the behavior of cows during of summer. Vet. Rec. 132:578-580.
Hull B.L., Weaver A.D., Koenig G.J., Welker B., Rings D.M., St Jean G., Crawford W.H., Tulleners E. \& Ames N.K. 1993. Diseases of the musculoskeletal system, p.864-881. In: Howard J.L. (Ed.), Current Veterinary Therapy - Food Animal Practice. $3^{\text {rd }}$ ed. W.B. Saunders, Philadelphia.

Martins C.F., Sarti E., Busato I., Pires P.P., Fiori C.H., Moreira C., Soares K., Betini B. \& Velasquez M. 2002. Prevalência e classificação das afecções podais em vacas lactantes na bacia leiteira de Campo Grande (Capital) e municípios arredores, MS. Ensaios e Ciências 6(2):113-137.

Melendez P., Bartolone J. \& Donavan A. 2002. Relationship between lameness, ovarian cysts and fertility in Holstein cows. Annals $12^{\text {th }}$ International Symposium on Disorders of the Ruminant Digit, Orlando, Florida, p.339-342.

Molina L.R., Carvalho A.U., Facury Filho E.J., Ferreira P.M. \& Ferreira V.C.P. 1999. Prevalência e classificação das afecções podais em vacas lactantes na bacia leiteira de Belo Horizonte. Arq. Bras. Med. Vet. Zootec. 51(2):149-152.

Moraes R.R. 2000. Caracterização clínica, laboratorial e anatomopatológica da fase inicial da inflamação do tecido interdigital de bovinos da raça Girolanda. Dissertação de Mestrado em Medicina Veterinária, Universidade Federal de Goiás, Goiânia, GO. 110p.

Silva L.A.F. 2001. Características clínicas e epidemiológicas das enfermidades podais em vacas lactantes do município de Orizona, GO. Ciênc. Anim. Bras. 2(2):119-126.

Silveira J.A.S., Albernaz T.T., Sousa M.G.S., Campos K.F., Silva N.S., Oliveira C.M.C., Duarte M.D., Barbosa J.D. 2008. Prevalência e características clínicas das enfermidades podais diagnosticadas pela central de diagnóstico veterinário (Cedivet), no estado do Pará, durante o período de 2000 a 2006. Encontro Nacional de Diagnóstico Veterinário, Campo Grande, MS, p.79-80. (Resumo expandido)

Toussaint Raven E. 1985. Cattle Footcare and Claw Trimming. Farming, Suffolk, p.20-63.

Venter B.J. \& van Amstel S.R. 1994. Fusobacterium necrophorum, Dichelobacter (Bacteroides) nodosus and Bacteroids spp. infections, p.1200-1228. In: Coetzer J.A.W., Thomson G.R. \& Tustin R.C. (Eds), Infectious Diseases of Livestock with special reference to Southern Africa. Oxford University Press, Cape Town.

Weaver A.D., Anderson L. \& De Laister-Banting A. 1981. Review of disorders of the ruminant digit with proposal for anatomical and pathological terminology and recording. Vet. Rec. 108:117-120.

Welker B. 1993. Interdigital fibroma, p.871-872. In: Howard J.L. (Ed.), Current Veterinary Therapy - Food Animal Practice. $3^{\text {rd }}$ ed. W.B. Saunders, Philadelphia. 